\title{
Poluição atmosférica e doenças respiratórias: um estudo de caso em Paranaguá, Paraná
}

Este trabalho apresenta uma avaliação da qualidade do ar da cidade que abriga o maior porto graneleiro do Brasil, Paranaguá/PR, e a sua relação com registros de doenças respiratórias nas unidades de saúde da cidade. O intenso tráfego de veículos que transportam cargas, faz dessa fonte uma das principais emissoras de poluentes atmosféricos em Paranaguá. As concentrações de dióxido de enxofre, dióxido de nitrogênio, monóxido de carbono, ozônio, materiais particulados totais e materiais particulados com até 10 micrômetros de diâmetro aerodinâmico (PM10) foram monitoradas de maio de 2016 a fevereiro de 2017 em uma estação de qualidade do ar distante em 200 metros de uma das principais vias de acesso ao porto. Desses poluentes, a concentração de PM10 ultrapassou o seu padrão de qualidade do ar em vigência no Brasil em $12 \%$ dos dias monitorados. A sua concentração variou com o tráfego de veículos na cidade, com maiores concentrações de dia e nos dias úteis. Esses materiais podem ser oriundos tanto da queima dos combustíveis quanto da dispersão da poeira das cargas transportadas pelos veículos. Uma vez que as vias de acesso ao porto de Paranaguá passam por regiões residenciais da cidade, as concentrações de PM10 medidas na estação de qualidade do ar também podem estar ocorrendo nessas regiões. A exposição contínua da população de Paranaguá a esses poluentes pode estar aumentando o número de casos de doenças respiratórias na cidade. A relação entre o número de casos de doenças respiratórias e a quantidade de habitantes é maior em Paranaguá do que em cidades mais urbanizadas, mais populosas e tipicamente mais frias.

Palavras-chave: Emissões atmosféricas; Doenças respiratórias; Qualidade do ar; Cidade portuária.

\section{Air pollution and respiratory diseases: a case study in Paranaguá, Paraná}

\begin{abstract}
This work presents an evaluation of the air quality of the city that houses the largest bulk port in Brazil, Paranaguá/PR, and its relationship with respiratory disease registers in the city's health units. The intense traffic of vehicles carrying cargo makes this source one of the main emitters of air pollutants in Paranaguá. The concentrations of sulfur dioxide, nitrogen dioxide, carbon monoxide, ozone, total particulate matter and particulate matter up to 10 micrometers in aerodynamic diameter (PM10) were monitored from May 2016 to February 2017 at an air quality station 200 meters away from one of the main access roads to the port. The concentration of PM10 exceeded its air quality standard in force in Brazil on $12 \%$ of the monitored days. PM10 concentration varied with vehicle traffic in the city, with higher rates during the day and during the week. These materials can come from burning fuels and dispersing dust from cargo carried by vehicles. As the access roads to the port of Paranaguá pass through residential regions of the city, the PM10 concentrations measured at the air quality station may also be occurring in these regions. The continuous exposure of the Paranaguá population to these pollutants may be increasing the number of cases of respiratory diseases in the city. The relationship between the number of respiratory diseases cases and the number of inhabitants is greater in Paranaguá than in more urbanized, more populous and typically colder cities.
\end{abstract}

Keywords: Atmospheric emissions; Respiratory diseases; Air quality; Port city.

Topic: Engenharia Ambiental

Reviewed anonymously in the process of blind peer

Gabriela Pereira Santana (iv)

Universidade Federal do Paraná, Brasil

http://lattes.cnpq.br/5472460830275586

http://orcid.org/0000-0002-6548-2667

1996santana@gmail.com

Virnei Silva Moreira (iD

Universidade Federal do Paraná, Brasil

http://lattes.cnpq.br/4964095231009486

http://orcid.org/0000-0001-9786-0469

virneimoreira@gmail.com

Fernando Augusto Silveira Armani id

Universidade Federal do Paraná, Brasil

http://lattes.cnpq.br/4870174841725558

http://orcid.org/0000-0001-9942-0555

fernando.armani@ufpr.br
Received: 05/08/2020

Approved: 26/09/2020

\section{Referencing this:}

SANTANA, G. P.; MOREIRA, V. S.; ARMANI, F. A. S.. Poluição atmosférica e doenças respiratórias: um estudo de caso em Paranaguá, Paraná. Revista Ibero Americana de Ciências Ambientais, v.11, n.5, p.352-361, 2020. DOI: http://doi.org/10.6008/CBPC2179$\underline{6858.2020 .005 .0033}$

DOI: 10.6008/CBPC2179-6858.2020.005.0033 


\section{INTRODUÇÃO}

O processo de urbanização mal planejado geralmente deteriora a qualidade do ar, pois concentra fontes de poluentes atmosféricos, e muitas vezes em locais desfavoráveis à dispersão dos mesmos (ALVES et al., 2009). Especificamente em cidades portuárias, o trânsito de veículos movidos à diesel é bastante intenso, consequentemente, nas proximidades das vias de tráfego, a concentração de poluentes atmosféricos é significativa (HABERT et al., 2015). Isso pode ser um problema em cidades cujo acesso à zona portuária passa por zonas residenciais, tal como em Paranaguá, município localizado no litoral do Paraná.

Atualmente, é amplamente sabido que a poluição atmosférica pode gerar e intensificar doenças no trato respiratório (CHANG et al., 2003; WILSON et al., 2005; MEDINA-RAMÓN et al., 2006; TAO et al., 2014). De acordo com a Organização Mundial da Saúde (OMS), todos os anos morrem cerca de sete milhões de pessoas por causas ligadas à poluição atmosférica, pois os níveis de poluição são elevados em diversas regiões do mundo (WHO, 2018). Assim como mencionado em Arbex et al. (2012), a OMS estimou que a poluição atmosférica é responsável por cerca de 20 mil óbitos por ano no Brasil.

Entre os principais poluentes atmosféricos estão: Materiais Particulados, Ozônio, Dióxido de Enxofre, Monóxido de Carbono e os Óxidos Nitrosos. Os efeitos desses poluentes na saúde humana são diversos. Os materiais particulados, por exemplo, podem obstruir e provocar irritação das vias aéreas. Aqueles com diâmetro inferior a 10 micrômetros, denominados $\mathrm{PM}_{10}$, podem alcançar os alvéolos pulmonares causando inflamação (BRAGA et al., 2001; WILSON et al., 2005). Já o ozônio e os óxidos nitrosos, o efeito mais comum desses gases é a irritação e a inflamação do trato respiratório (ARBEX et al., 2012). Segundo Martins et al. (2002), o $\mathrm{SO}_{2}$ é um gás muito solúvel com alta taxa de absorção nas vias aéreas; entre as consequências negativas da exposição a este gás é o decréscimo da função pulmonar, e a redução da imunidade, deixando o indivíduo suscetível à pneumonia e a gripe. Em ambientes interiores, um dos poluentes mais preocupantes é o Monóxido de Carbono, pois esse gás é inodoro e asfixiante (BRANCO et al., 2010). A exposição contínua a esses poluentes atmosféricos pode gerar doenças respiratórias, tal como a bronquite (BRAGA et al., 2001) e a asma (CANÇADO et al., 2006), aumentar a susceptibilidade às doenças infecciosas, como a gripe, e consequentemente, aumentar o nível de mortalidade e de internações hospitalares (KAN et al., 2007; LEE et al., 2000; YANG et al., 2004; ZEKA et al., 2005).

Uma vez que os poluentes atmosféricos interferem na saúde humana, foram definidas concentrações limites para esses fluidos na atmosfera, para garantir a saúde e o bem-estar da população (LISBOA, 2007). Essas concentrações são denominadas padrões de qualidade do ar e estão regulamentadas na Resolução CONAMA no 491 de 2018. Neste trabalho, comparou-se as concentrações dos gases $\mathrm{O}_{3}, \mathrm{NO}_{\mathrm{x}}, \mathrm{SO}_{2}, \mathrm{PM}_{10}$ e CO na atmosfera de Paranaguá com os seus padrões de qualidade do ar, e a relação entre a concentração de alguns desses poluentes com registros de casos das seguintes doenças respiratórias: pneumonia, gripe, asma e bronquite. 


\section{METODOLOGIA}

O presente estudo foi realizado no município de Paranaguá, que está localizado entre as latitudes $25,365^{\circ} \mathrm{S}$ e $25,735^{\circ} \mathrm{S}$, e longitudes $48,706^{\circ} \mathrm{W}$ e $48,277^{\circ} \mathrm{W}$, no litoral do estado do Paraná (vide o mapa da Figura 1).

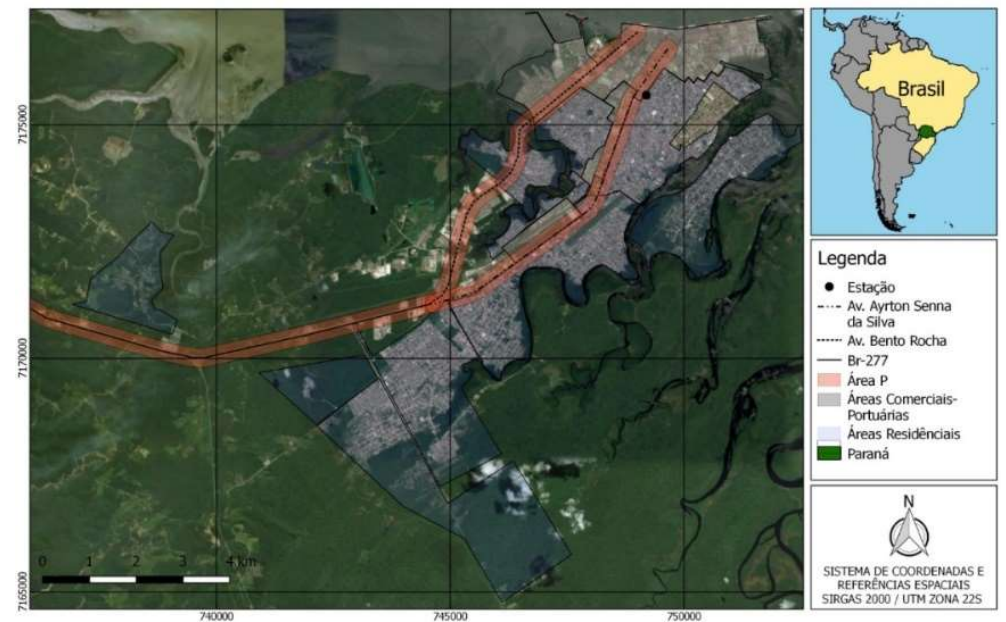

Figura 1: Mapa da cidade de Paranaguá, com destaque para as áreas residenciais da cidade, área comercial portuária e para a faixa de poluição das avenidas de acesso ao Porto de Paranaguá (Área P).

Em Paranaguá está o maior porto graneleiro do Brasil. Logo, há um intenso tráfego de caminhões nas principais vias de acesso ao porto, assim como grande movimentação de trens e navios: o cais do porto suporta 14 navios. Na cidade também há indústrias de alimentos e fertilizantes, silos de grãos, tanques de álcool, entre outras fontes diretas e indiretas de poluentes atmosféricos associadas ao Porto. Com base na lei municipal no 62/2007, a qual estabelece o zoneamento de uso e ocupação do solo do município (PARANAGUÁ, 2007), as áreas comerciais portuárias foram destacadas no mapa da Figura 1 na coloração cinza e as áreas residenciais foram destacadas na coloração azul.

Este trabalho apresenta uma avaliação da qualidade do ar da região urbanizada de Paranaguá e a sua relação com doenças respiratórias. Os dados de qualidade do ar foram obtidos com o IAP (Instituto Ambiental do Paraná), órgão ambiental do estado do Paraná responsável pelo monitoramento da qualidade do ar na cidade. Os dados são de maio de 2016 a fevereiro de 2017, e foram medidos por uma estação de monitoramento instalada na sede da Secretaria de Obras da prefeitura de Paranaguá, nas coordenadas: latitude de $25,515432^{\circ} \mathrm{S}$ e longitude $48,520516^{\circ} \mathrm{W}$. Como se pode observar na Figura 1 , a estação está localizada a cerca de $\mathbf{2 0 0}$ metros de distância da Avenida Ayrton Senna da Silva, uma das principais vias de acesso ao porto, e à aproximadamente $1 \mathrm{~km}$ de distância da Avenida Bento Rocha, principal via de acesso ao pátio de caminhões de Paranaguá. A distância de 200 metros da avenida Ayrton Senna da Silva à estação foi utilizada para definir a espessura de uma faixa de poluição do ar (Área P no mapa) gerada pelo tráfego dos veículos nas avenidas, sob a hipótese de que as concentrações dos poluentes nessa faixa são iguais ou maior ao que foi monitorado na estação do Instituto Ambiental do Paraná: quanto mais próximo do local da fonte de poluição (vias de acesso ao porto), maior é a concentração dos poluentes.

A estação realiza medições horárias de poluentes atmosféricos e variáveis meteorológicas. Os 
poluentes monitorados são: Ozônio $\left(\mathrm{O}_{3}\right)$, Materiais particulados totais em suspensão (PTS), partículas inaláveis $\left(\mathrm{PM}_{10}\right)$, monóxido de carbono $(\mathrm{CO})$, monóxido de nitrogênio $(\mathrm{NO})$, dióxido de nitrogênio $\left(\mathrm{NO}_{2}\right)$, óxidos de nitrogênio $\left(\mathrm{NO}_{\mathrm{x}}\right)$, dióxido de enxofre $\left(\mathrm{SO}_{2}\right)$, metano $\left(\mathrm{CH}_{4}\right)$ e hidrocarbonetos não metanos (NMHC). As variáveis meteorológicas monitoradas são: umidade relativa do ar, precipitação, intensidade do vento, direção do vento, pressão barométrica, temperatura e radiação solar. A estação foi instalada em maio de 2016. Segundo o fabricante, o prazo de calibração de todos os sensores é de 1 ano: período do monitoramento cujos dados estão apresentados neste trabalho.

Durante o período de monitoramento ocorreram interrupções nas medições da estação de qualidade do ar devido à queda de energia ou ao período de manutenção da estação, e também foram identificados 'outliers' e períodos com interrupções em parte dos analisadores da estação. Para garantir a qualidade das medições, removeu-se os 'outliers' (identificados pelos valores superiores ao limite de detecção dos sensores) e avaliou-se apenas os dias com mais de $80 \%$ das horas medidas. Com isso, dos 10 meses monitorados foram avaliados 217 dias neste trabalho.

Os casos de internações mensais por pneumonia e gripe foram obtidos através do site do Departamento de Informática do Sistema Único de Saúde (DATASUS). Como a pneumonia e a gripe encontram-se no mesmo grupamento do Código Internacional de Doenças (CID 10), foram consideradas uma só neste trabalho. Os dados mensais de diagnósticos de asma e bronquite foram disponibilizados pela Secretaria Municipal de Saúde de Paranaguá, ambas estão sendo avaliadas em conjunto neste trabalho com a denominação de doenças crônicas.

Para a avaliação da qualidade do ar de Paranaguá, compararam-se as concentrações dos poluentes com os padrões finais de qualidade do ar estabelecidos na Resolução CONAMA no 491 de 2018. Na Tabela 1 foram dispostos os limites de concentração de poluentes atmosféricos propostos pela OMS, que foram padronizados na Resolução CONAMA 491/18 como Padrão Final e as maiores concentrações dos poluentes atmosféricos monitorados na estação do IAP durante o período avaliado neste trabalho.

Tabela 1: Padrões de concentração estabelecidos na Resolução CONAMA 491 de 2018 e as maiores concentrações medidas pela estação do IAP nos anos de 2016 e 2017.

\begin{tabular}{l|l|l|l|}
\hline Poluente & $\begin{array}{l}\text { Período de } \\
\text { referência }\end{array}$ & Concentração Padrão & $\begin{array}{l}\text { Maior concentração monitorada } \\
\text { em Paranaguá }\end{array}$ \\
\hline $\mathrm{NO}_{2}$ & Média horária & $200 \mu \mathrm{g} / \mathrm{m}^{3}$ & $53,96 \mu \mathrm{g} / \mathrm{m}^{3}$ \\
\hline $\mathrm{CO}$ & Média móvel (8 horas) & $9 \mathrm{ppm}$ & $1,39 \mathrm{ppm}$ \\
\hline $\mathrm{O}_{3}$ & Média móvel (8 horas) & $100 \mu \mathrm{g} / \mathrm{m}^{3}$ & $43,91 \mu \mathrm{g} / \mathrm{m}^{3}$ \\
\hline $\mathrm{SO}_{2}$ & Média diária & $20 \mu \mathrm{g} / \mathrm{m}^{3}$ & $17,21 \mu \mathrm{gg} / \mathrm{m}^{3}$ \\
\hline $\mathrm{PTS}$ & Média diária & $240 \mu \mathrm{g} / \mathrm{m}^{3}$ & $189,26 \mu \mathrm{gg} / \mathrm{m}^{3}$ \\
\hline $\mathrm{PM}_{10}$ & Média diária & $50 \mu \mathrm{g} / \mathrm{m}^{3}$ & $84,00 \mu \mathrm{g} / \mathrm{m}^{3}$ \\
\hline
\end{tabular}

\section{RESULTADOS E DISCUSSÃO}

Em Paranaguá, a principal fonte de poluição do ar é a emissão de veículos movidos à diesel que transportam cargas. Entre os poluentes atmosféricos mais emitidos por esses veículos estão os materiais particulados (GAFFNEY et al., 2009), tal como o $\mathrm{PM}_{10}$, que tiveram concentrações superiores ao padrão de 
qualidade do ar durante o período de monitoramento avaliado neste trabalho (vide Tabela 1). No total, em 26 dias a concentração de $\mathrm{PM}_{10}$ ultrapassou seu padrão final de qualidade do ar. A Figura 2 exibe a quantidade de ocorrências desses episódios nos meses avaliados neste trabalho e as maiores concentrações médias de $24 \mathrm{~h}$ obtidas em cada um desses meses.

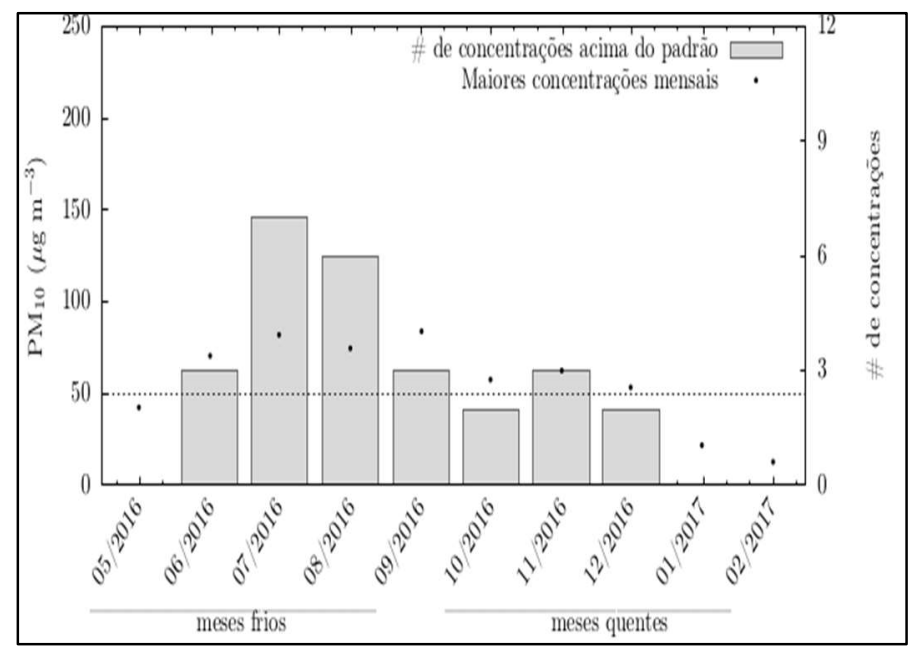

Figura 2: Variação das maiores concentrações de PM10 no período de monitoramento apresentado neste trabalho. As barras indicam as quantidades (\#) mensais de concentrações de $\mathrm{PM}_{10}$ que superaram seu padrão de qualidade do ar, os pontos pretos são as maiores concentrações medidas em cada um dos meses e a linha pontilhada indica a concentração padronizada na Resolução Conama 491/2018 para PM10.

Nota-se na Figura 2, que tanto nos meses frios quanto nos meses quentes foram obtidas concentrações médias diárias de $\mathrm{PM}_{10}$ acima do padrão de qualidade do ar estabelecido na resolução. Em aproximadamente $12 \%$ dos 217 dias monitorados, as concentrações de $\mathrm{PM}_{10}$ estavam num nível superior àquele determinado como Padrão Final na Resolução Conama 491 de 2018.

Devido às atividades portuárias, o fluxo de veículos é mais intenso de segunda à sexta-feira, consequentemente as concentrações dos poluentes atmosféricos são maiores nesses dias: 96\% das concentrações médias diárias de $\mathrm{PM}_{10}$ que ultrapassaram os seus padrões finais foram obtidas nesses dias. Quanto à variação das concentrações de materiais particulados na atmosfera ao longo do dia, também corresponde ao tráfego de veículos na cidade. Embora o porto de Paranaguá opere durante todo o dia, o tráfego de veículos é mais intenso no período diurno, com as vias de acesso ao porto geralmente congestionadas até às $20 \mathrm{~h}$. Logo, $71 \%$ das maiores concentrações horárias (superiores a $50 \mu \mathrm{g} \mathrm{m}^{-3}$ ) foram medidas entre $6 \mathrm{~h}$ e $20 \mathrm{~h}$.

A principal fonte dos poluentes atmosféricos medidos na estação do IAP é provavelmente a emissão veicular, tanto no processo de queima de combustível quanto na dispersão da poeira das cargas transportadas; logo, as mesmas concentrações dos poluentes medidos na estação do IAP podem estar ocorrendo na faixa que contorna as avenidas de acesso ao porto destacada na Figura 1 como Área P. Como se pode notar na Figura 1, essa faixa atravessa uma grande parte das áreas residenciais da cidade, submetendo a população aos poluentes emitidos por esses veículos.

A Figura 3 exibe os gráficos de dispersão das concentrações médias diárias de PTS contra PM $_{10}$ nos meses frios e quentes. Nota-se tanto na Figura 2 quanto na Figura 3, que as concentrações médias diárias 
superiores ao padrão de qualidade do ar foram mais frequentes nos meses frios e as maiores concentrações de $\mathrm{PM}_{10}$ e PTS foram medidas nesses meses. Observa-se no coeficiente angular da regressão linear da Figura 3, que a fração de materiais particulados finos $\left(\mathrm{PM}_{10}\right)$ nos materiais particulados totais é aproximadamente igual nos meses frios e nos meses quentes e que a concentração de $\mathrm{PM}_{10}$ na atmosfera é geralmente em torno de $50 \%$ da concentração de PTS. Essa fração mostra que a fonte de material particulado à atmosfera local emite materiais particulados finos em abundância, tal como os emitidos por veículos automotores (RISTOVSKI et al., 2012).

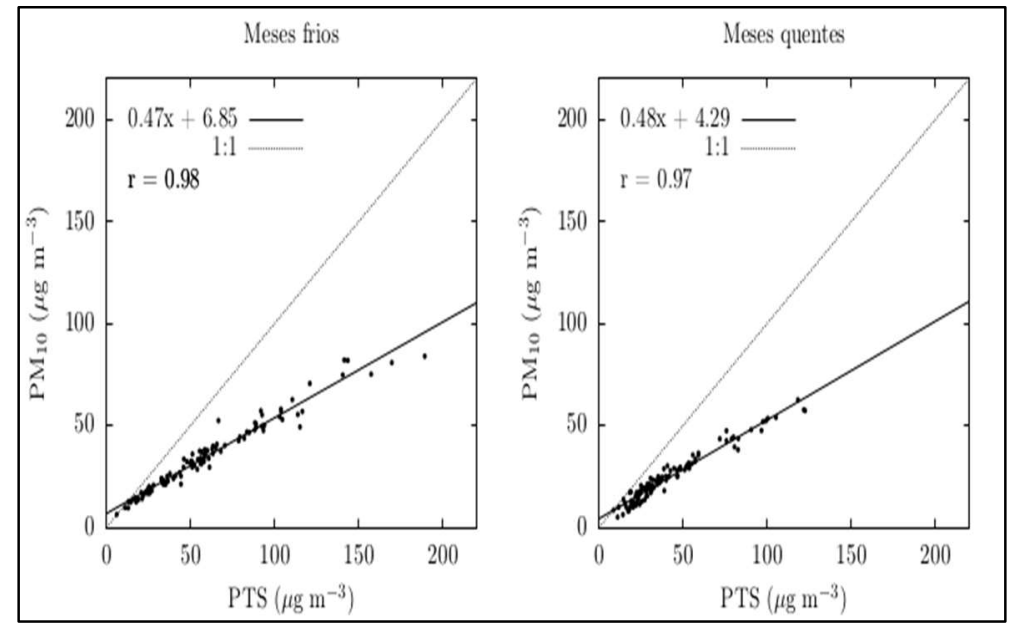

Figura 3: Gráfico de dispersão das concentrações horárias de PM10 e PTS.

A forte associação entre PTS e PM10 identificada pelo coeficiente de correlação de Pearson neste trabalho, é comumente reportada na literatura (GÓMEZ et al., 2003; MONN et al., 1995; SALDARRIAGA et al., 2004; CANDANOZA et al., 2013): quanto maior a concentração de materiais particulados totais na atmosfera, maior é a concentração de partículas finas inaláveis.

Pan et al. (2010) mostraram em um estudo realizado em 6 cidades intensamente urbanizadas do Norte da China, que a concentração de materiais particulados na atmosfera também tem uma forte associação com problemas respiratórios em crianças, principalmente asma, chiado no peito e tosse persistente. Em geral, as doenças respiratórias avaliadas neste trabalho são intensificadas em atmosferas poluídas por materiais particulados emitidos por motores à diesel (RISTOVSKI et al. 2012; HABERT et al., 2015).

As concentrações dos poluentes atmosféricos são geralmente maiores nos períodos mais frios (meses frios e à noite), pois a capacidade de dispersão dos poluentes pela turbulência atmosférica é reduzida neste período (TORRES et al., 2005). Logo, mais casos de internações por doenças respiratórias ou diagnósticos de asma ou bronquite são registrados (ZHU et al., 2012). O percentual de ocorrências de atendimentos hospitalares envolvendo as doenças respiratórias avaliadas neste trabalho foi $82 \%$ maior nos meses frios em relação aos meses quentes. De acordo com Rullo et al. (2018), o ar frio também é um forte estímulo a crises de asma e bronquite.

No período avaliado neste trabalho, em Paranaguá foram contabilizados 635 casos de internações por pneumonia, 8 casos de internações por gripe, 6 pacientes foram diagnosticados com asma e 29 foram 
diagnosticados com bronquite. As poucas internações por gripe são por conta das campanhas de vacinação, que são gratuitas no país. O Brasil vem se prevenindo de uma epidemia da gripe através da vigilância do vírus e seus subtipos pelo Ministério da Saúde (FUCHS, 2018). Já as poucas ocorrências de registros de asma em Paranaguá podem ser devido à dificuldade em ser diagnosticada, pois os sintomas são semelhantes à de outras doenças (FONTES et al., 2005).

A Figura 4 exibe as quantidades mensais de registros hospitalares de casos de doenças respiratórias em Paranaguá e as quantidades mensais das concentrações médias diárias de $\mathrm{PM}_{10}$ que ultrapassaram os padrões de qualidade do ar. Como é de se esperar, nos meses mais frios, em Paranaguá, foram registrados mais casos de doenças respiratórias e mais concentrações de $\mathrm{PM}_{10}$ ultrapassaram o padrão de qualidade do ar.

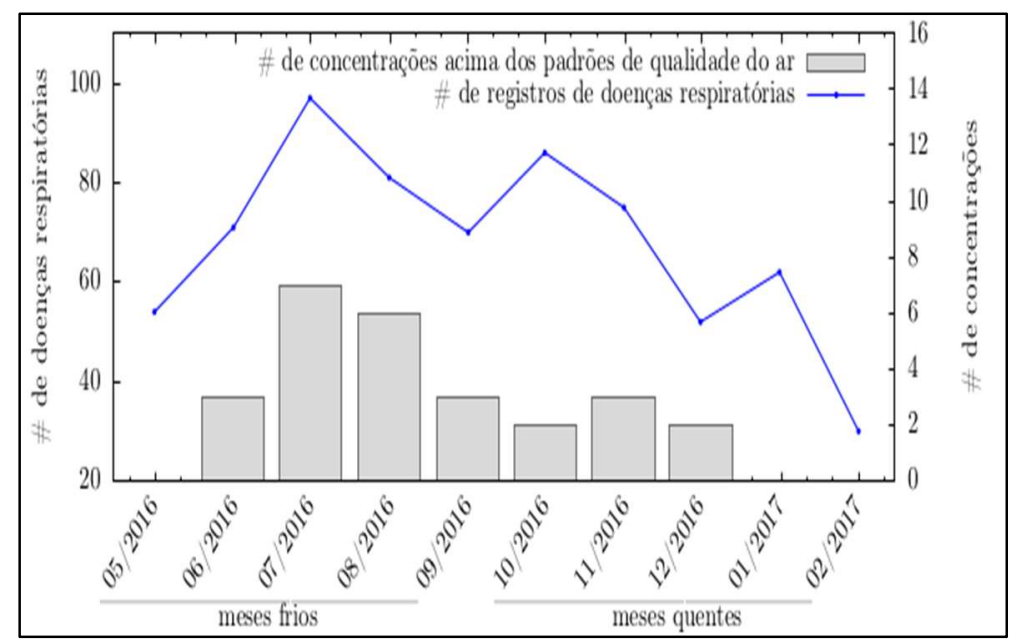

Figura 4: Quantidade (\#) de registros de atendimentos de doenças respiratórias e quantidade de concentrações dos poluentes que ultrapassaram os padrões de qualidade do ar estabelecidos na Resolução Conama 491/18 no período de monitoramento avaliado.

A quantidade de doenças respiratórias em Paranaguá em relação à sua população é maior do que em cidades tipicamente mais frias e mais urbanizadas. Embora esse índice possa ser influenciado por outras variáveis, é um indicador razoável das consequências da qualidade do ar à saúde dos habitantes da cidade. $\mathrm{Na}$ Tabela 2 estão listadas algumas cidades brasileiras e as quantidades de registros de doenças respiratórias no mesmo período avaliado neste trabalho.

Tabela 2: Número de casos de internações por pneumonia e gripe, e pacientes diagnosticados com asma e bronquite em municípios brasileiros, durante o período de maio de 2016 a fevereiro de 2017 (Datasus). As temperaturas são as máximas e mínimas climatológicas (INMET) e a população é a estimada para o ano de 2019 pelo IBGE.

\begin{tabular}{|l|l|l|l|l|l|}
\hline Município - UF & População & Temp. máxima $\left({ }^{\circ} \mathrm{C}\right)$ & Temp. mínima $\left({ }^{\circ} \mathrm{C}\right)$ & $\mathrm{N}^{\circ}$ de casos & $\mathrm{N}^{\circ}$ de casos/população \\
\hline Paranaguá - PR & 154.936 & 30 & 22 & 686 & 0,00443 \\
\hline Rio Grande - RS & 211.005 & 27 & 16 & 807 & 0,00382 \\
\hline Ponta Grossa - PR & 351.736 & 28 & 21 & 1336 & 0,00380 \\
\hline Navegantes - SC & 81.475 & 27 & 19 & 309 & 0,00379 \\
\hline Maringá - PR & 423.666 & 31 & 24 & 977 & 0,00231 \\
\hline Itajaí - SC & 219.536 & 27 & 19 & 486 & 0,00221 \\
\hline Cascavel - PR & 328.454 & 29 & 21 & 516 & 0,00157 \\
\hline Cubatão - SP & 130.705 & 26 & 21 & 120 & 0,00092 \\
\hline
\end{tabular}


Observa-se na Tabela 2, que Paranaguá tem mais casos de doenças respiratórias por quantidade de habitantes do que em todas as outras cidades listadas na tabela, inclusive na cidade de Cubatão/SP. Cubatão é um dos maiores polos industriais do país. Na década de 1980, Cubatão foi considerada a cidade mais poluída do mundo (ALONSO et al., 1992). Apesar disso, com as ações ambientais que estimularam a melhoria da qualidade do ar e do meio ambiente, atualmente Cubatão tem menos registros de doenças respiratórias e há trabalhos relatando que as concentrações na atmosfera da cidade melhoraram significativamente. Por exemplo, Nardocci et al. (2013) avaliaram a concentração de PM10 em Cubatão durante 5 anos e não constataram concentrações maiores do que a concentração padrão de qualidade do ar de $50 \mu \mathrm{g} / \mathrm{m}^{3}$.

\section{CONCLUSÕES}

A poluição do ar ambiente é reconhecida como um problema importante, nacional e mundialmente. A compreensão científica dos efeitos da poluição do ar na saúde aumentou, bem como os estudos que reportam o impacto da poluição do ar na qualidade de vida das pessoas. Neste contexto, este trabalho apresenta uma avaliação da qualidade do ar da cidade de Paranaguá, Estado do Paraná, e a sua relação com os casos de doenças respiratórias na cidade. $O$ estudo é baseado em medições de poluentes atmosféricos realizadas por uma estação automática do Instituto Ambiental do Paraná e por registros de doenças respiratórias disponíveis no sistema Datasus e na secretaria de saúde do município de Paranaguá. Os dados avaliados são do período de maio de 2016 a fevereiro de 2017.

Na cidade de Paranaguá há um intenso tráfego de veículos movidos à diesel transportando cargas, pois nela está o maior porto graneleiro do Brasil. Os poluentes atmosféricos são emitidos por esses veículos tanto na queima de combustível quanto na dispersão do pó dos produtos transportados. Logo, a principal fonte de poluentes atmosféricos em Paranaguá é a veicular, de modo que as concentrações dos poluentes medidos na estação de qualidade do ar do IAP variaram com o tráfego de veículos na cidade.

Entre os poluentes avaliados neste trabalho $\left(\mathrm{SO}_{2}, \mathrm{NO}_{2}, \mathrm{CO}, \mathrm{O}_{3}, \mathrm{PTS}\right.$ e $\left.\mathrm{PM}_{10}\right)$, a concentração de $\mathrm{PM}_{10}$ ultrapassou o seu padrão de qualidade do ar estabelecido na legislação brasileira em $12 \%$ dos 217 dias avaliados. Considerando que a principal fonte desse poluente em Paranaguá é a veicular, esse cenário é agravado porque as vias de acesso ao porto passam por grande parte das áreas residenciais e comerciais da cidade.

As altas concentrações de materiais particulados finos na atmosfera são preocupantes, pois essas partículas menores representam um risco maior à saúde, principalmente nas populações mais vulneráveis (crianças e idosos). Consequentemente, a poluição atmosférica em Paranaguá pode estar intensificando os casos de doenças respiratórias, pois se constatou neste trabalho que a quantidade de casos de doenças respiratórias em Paranaguá em relação ao número de habitantes é maior do que em cidades mais populosas, mais urbanizadas e tipicamente mais frias.

AGRADECIMENTOS: Agradecemos ao Instituto Ambiental do Paraná pela disponibilização de seus dados. 


\section{REFERÊNCIAS}

ALONSO, C. D.; GODINHO, R.. A evolução da qualidade do ar em Cubatão. Química Nova, v.15, n.2, p.126-136, 1992.

ALVES, K. M. S.; ALVES, A. E. L.; SILVA, F. M.. Poluição do ar e saúde nos principais centros comerciais da cidade de Natal/RN. Holos, v.4, p.80-95, 2009.

ARBEX, M. A.; SANTOS, U. P.; MARTINS, L. C.; SALDIVA, P. H. N.; PEREIRA, L. A. A.; BRAGA, A. L. F.. A poluição do ar e o sistema respiratório. Jornal Brasileiro de Pneumologia, v.38, n.5, p.643-655, 2012.

BRAGA, A.; BÖHM, G. M.; PEREIRA, L. A. A.; SALDIVA, P.. Poluição atmosférica e saúde humana. Revista USP, n.51, p.58-71, São Paulo, 2001.

BRANCO, S. M.; MURGEL, E.. Poluição do ar. Moderna, v.1, n. 2,2010

CANÇADO, J. E. D.; BRAGA, A.; PEREIRA, L. A. A.; ARBEX, M. A.; SALDIVA, P. H. N.; SANTOS, U. P.. Repercussões clínicas da exposição à poluição atmosférica. Jornal Brasileiro de Pneumologia, v.32, p.5-11, 2006.

CANDANOZA, S.; GORIBAR, L.; GARCÍA, F.. Relación particulas respirables $\left(\mathrm{PM}_{10}\right) /$ particulas suspendidas totales (PST) en Santa Marta (Colombia). Dyna. v.80, n.179, p.157164, 2013.

CHANG, G. Q.; WANG, L. G.; PAN, X. C.. Study on the associations between ambient air pollutant and hospital outpatient visit or emergency room visit in Beijing. China School Doctor, v.17, n.4, p.295-297, 2003

FONTES, M. J. F.; FONSECA, M. T. M.; CAMARGOS, P. A. M.; AFFONSO, A. G. A.; CALAZANS, G. M. C.. Asthma in children under five years of age: problems in diagnosis and in inhaled corticosteroid treatment. Jornal Brasileiro de Pneumologia. v.31, n.3, p.244-253, 2005.

FUCHS, A.. Influenza: conheça os subtipos existentes e saiba como evitar a contaminação. Rio de Janeiro: Fundação Oswaldo Cruz, 2018.

GAFFNEY, J. S.; MARLEY, A. M.. The impacts of combustion emissions on air quality and climate: From coal to biofuels and beyond. Atmospheric Environment, v.43, p.23-36, 2009. DOI: https://doi.org/10.1016/j.atmosenv.2008.09.016

GÓMEZ, A.; HENAO, Y.; MOLINA, F.; MOLINA, F.. Evaluación de las partículas suspendidas totales (PST) y partículas respirables $\left(\mathrm{PM}_{10}\right)$ en la zona de Guayabal, Medellín, Colombia. Revista Facultad de Ingeniería, n.30, p.24- 33, 2003.

HABERT, C.; GARNIER, R.. Effets sur la santé des émissions des moteurs diesel: revue des connaissances. Rev. Mal.

Respir, v.32, p.138-154, 2015. DOI:

http://dx.doi.org/10.1016/j.rmr.2014.07.012

KAN, H.; LONDON, S. J.; CHEN, G.; ZHANG, Y.; SONG, G.; ZHAO, N.; JIANG, L.; CHEN, B.. Differentiating the effects of fine and coarse particles on daily mortality in Shanghai, China. Environ. Int., v.33, n.3, p.376-384, 2007. DOI: https://doi.org/10.1016/i.envint.2006.12.001
LEE, J. T.; KIM, H.; HONG, Y. C.; KWON, H. J.; SCHWARTZ, J.; CHRISTIANI, D. C.. Air pollution and daily mortality in seven major cities of Korea, 1991 e 1997. Environ. Res. A, v.84, n.3, p.247-254, 2000. DOI:

https://doi.org/10.1006/enrs.2000.4096

LISBOA, H. M.. Meteorologia e dispersão atmosférica. Montreal, 2007.

MARTINS, L. C.; LATORRE, M. R. D. O.; CARDOSO, M. R. A.; GONÇALVES, F. L. T.; SALDIVA, P. H. N.; BRAGA, A. L. F.. Poluição atmosférica e atendimentos por pneumonia e gripe em São Paulo, Brasil. Revista de Saúde Pública, v.36, n.1, p.88-94, 2002.

MEDINA-RAMÓN, M.; ZANOBETTI, A.; SCHWARTZ, J.. The effect of ozone and $\mathrm{PM}_{10}$ on hospital admissions for pneumonia and chronic obstructive pulmonary disease: a National Multicity Study. Am. J. Epidemiol. v.163, n.6, p.579588, 2006. DOI: https://doiorg.ez22.periodicos.capes.gov.br/10.1093/aje/kwj078

MONN, C. H.; BRAENDLI, O.; SCHAEPPI, G.; ACKERMANNLIEBRICH, U.; LEUENBERGER, P. H.; TEAM, S.. Particulate matter $<10 \mu \mathrm{m}$ (PM10) and total suspended particulates (TSP) in urban, rural and alpine air in Switzerland. Atmospheric Environment, v.29, n.19, p.2565-2573, 1995. DOI: https://doi.org/10.1016/1352-2310(95)94999-U

NARDOCCI, A. C.; FREITAS, C. U.; LEON, A. C. M. P.; JUNGER, W. L.; GOUVEIA, N. C.. Poluição do ar e doenças respiratórias e cardiovasculares: estudo de séries temporais em Cubatão, São Paulo, Brasil. Cad. Saúde Pública, v.29, n.9, p.18671876, 2013. DOI: https://doi.org/10.1590/0102$311 \times 00150012$

PAN, G.; ZHANG, S.; FENG, Y.; TAKAHASHI, K.; KAGAWA, J.; YU, L.; WANG, P.; LIU, M.; LIU, Q.; HOU, S.; PAN, B.; LI, J.. Air pollution and children's respiratory symptoms in six cities of Northern China. Respiratory Medicine, v.104, p.1903-1911, 2010. DOI: https://doi.org/10.1016/j.rmed.2010.07.018

PARANAGUÁ. Lei complementar $\mathbf{n}$. 62, de $\mathbf{2 7}$ de agosto de 2007. Institui o zoneamento de uso e ocupação do solo do município de Paranaguá, e dá outras providências. Paranaguá: DOE, 2007.

RISTOVSKI, Z. D.; MILEVIC, B.; SURAWSKI, N. C.; MORAWSKA, L.; FONG, K. M.; GOH, F.; YANG, I. A.. Respiratory health effects of diesel particulate matter. Respiralogy, v.17, p.201-212, 2012.

DOI: https://doi.org/10.1111/j.1440-1843.2011.02109.x

RULLO, V.; SILVA, T. N.. Asma e bronquite não são a mesma coisa. Saúde Abril, 2018.

SALDARRIAGA, J. C. M.; ECHEVERRI, C. A. L.; MOLINA, F. J. P.. Partículas suspendidas (PST) y partículas respirables $\left(\mathrm{PM}_{10}\right)$ en el Valle de Aburrá, Colombia. Revista Facultad de Ingeniería, v.32, p.7-16, 2004.

TAO, Y.; MI, S.; ZHOU, S.; WANG, S.; XIE, X.. Air pollution and hospital admissions for respiratory diseases in Lanzhou, China. Environmental Pollution, v.185, p.196-201, 2014. 


\section{DOI: https://doi.org/10.1016/j.envpol.2013.10.035}

TORRES, F. T. P.; MARTINS, L. A.. Fatores que influenciam na concentração do material particulado inalável na cidade de Juiz de Fora (MG). Caminhos da Geografia, p.23-39, 2005.

WILSON, A. M.; WAKE, C. P.; KELLY, T.; SALLOWAY, J. C.. Air pollution, weather, and respiratory emergency room visits in two northern New England cities: an ecological time - series study. Environ. Res., v.97, n.3, p.312-321, 2005.

DOI: https://doi.org/10.1016/j.envres.2004.07.010

WILSON, J. G.; KINGHAM, S.; PEARCE, J.; STURMAN, A. P.. A review of intraurban variations in particulate air pollution: implications for epidemiological research. Atmospheric Environment, v.39, n.34, p.6444-6462, 2005.

DOI: https://doi.org/10.1016/j.atmosenv.2005.07.030

WHO. World Health Organization. 9 out 10 people worldwide breathe polluted air, but more countries are taking action. Genebra: WHO, 2018

YANG, C. Y.; CHANG, C. C.; CHUANG, H. Y.; TSAI, S. S.; WU, T. N.; HO, C. K.. Relationship between air pollution and daily mortality in a subtropical city: Taipei, Taiwan. Environ. Int., v.30, n.4, p.519-523, 2004. DOI:

https://doi.org/10.1016/j.envint.2003.10.006

ZEKA, A.; ZANOBETTI, A.; SCHWARTZ, J.. Short term effects of particulate matter on cause specific mortality: effects of lags and modification by city characteristics. Occup. Environ. Med., v.62, n.10, p.718-725, 2005.

DOI: http://dx.doi.org/10.1136/oem.2004.017012

ZHU, W.; WANG, J.; ZHANG, W.; SUN, D.. Short-term effects of air pollution on lower respiratory diseases and forecasting by the group method of data handling. Atmospheric Environment, v.51, p.29-38, 2012. DOI: https://doi.org/10.1016/j.atmosenv.2012.01.051

A CBPC - Companhia Brasileira de Produção Científica (CNPJ: 11.221.422/0001-03) detém os direitos materiais desta publicação. Os direitos referem-se à publicação do trabalho em qualquer parte do mundo, incluindo os direitos às renovações, expansões e disseminações da contribuição, bem como outros direitos subsidiários. Todos os trabalhos publicados eletronicamente poderão posteriormente ser publicados em coletâneas impressas sob coordenação da Sustenere Publishing, da Companhia Brasileira de Produção Científica e seus parceiros autorizados. Os (as) autores (as) preservam os direitos autorais, mas não têm permissão para a publicação da contribuição em outro meio, impresso ou digital, em português ou em tradução. 